\title{
Alternative Position and Navigation based on DME Accumulated Delta Range
}

\author{
Tunc Ertan and Mark L. Psiaki, \\ Cornell University, Ithaca, $N Y$
}

\section{BIOGRAPHIES}

Tunc Ertan is pursuing a Ph.D. in the Sibley School of Mechanical and Aerospace Engineering at Cornell University. He received a B.S. in Mechanical Engineering from Cornell University. His current research interests are in the areas of GNSS and alternative radio-navigation technologies, estimation, and filtering.

Mark L. Psiaki is a Professor of Mechanical and Aerospace Engineering. He received a B.A. in Physics and M.A. and $\mathrm{Ph} . \mathrm{D}$. degrees in Mechanical and Aerospace Engineering from Princeton University. His research interests are in the areas of GNSS and alternative radio-navigation technologies and applications, spacecraft attitude and orbit determination, and general estimation, filtering, and detection.

\section{ABSTRACT}

A method is analyzed that estimates absolute aircraft position and velocity based on time-series of accumulated delta range measurements from Distance Measuring Equipment (DME) ground transponders. This method provides an Alternative Position and Navigation - but not quite Timing - (APNT) system for use in airplanes as a backup to the Global Navigation Satellite System (GNSS). It performs absolute navigation based on relative-change-inrange measurements. These measurements are provided by nearly continuous radio-navigation carrier phase observables. A Square Root Information Extended Kalman Filter (SRIF) is developed for this method, and a corresponding medium-fidelity truth-model simulation is used to evaluate the filter performance in absolute position observability, filter convergence times, and steady state accuracies for representative scenarios. The results show that a steady state accuracy level below 0.025 nautical miles is achievable, and they reveal the potential usefulness of the proposed method as part of the Federal Aviation Administration's APNT program.

\section{INTRODUCTION}

Position, Navigation, and Timing (PNT) services are vital to many essential applications worldwide. In particular, the Global Navigation Satellite System (GNSS) has been increasingly adopted into civilian use, and GNSS will become the primary, and increasingly the only, system supporting navigation services for the civilian airspace. However, GNSS has been shown to be vulnerable to spoofing and jamming in recent years. In light of the vulnerability of GNSS, the Federal Aviation Administration (FAA) has started an Alternative Position, Navigation, and Timing (APNT) program that seeks to provide back-up navigation services in the National Airspace even in the event of GNSS service degradation or outage [1].

The use of the existing Distance Measuring Equipment (DME) ground transponder network has been proposed for this purpose. DME determines the slant range between an aircraft and the ground transponder by round trip two-way ranging. The total time from an aircraft radio interrogation to the arrival of a ground-transponder reply is measured and converted to a distance measurement. This process is visualized in Fig. 1 by the first item, in which the blue arrow represents the aircraft interrogation, and the red arrow stands for the transponder reply.

One of the proposed APNT methods is active aircraft interrogation of multiple DME transponders to achieve aircraft position. In this method, the aircraft interrogates all the DME transponders in view, and its flight management system filters the multiple range data to solve for its position. The use of existing infrastructure is the main attraction of this method. Because a DME transponder has to respond to interrogation by multiple aircraft in order for all of them to get a range measurement, this method has a system capacity limited by the response rate of the DME transponder and the fact that the DME transponder can only reply to one interrogation at a time. Therefore, the effective number of ground stations at an instant is reduced from its theoretical maximum of the total number of ground stations in view to a lower number. 
A second proposed method is DME-based passive ranging [2]. This method replaces the active interrogation of the previous method with on-ground pseudo interrogators. Each DME ground station transmits a response to its pseudo interrogator as if being interrogated by an aircraft. Unlike in the previous method, the DME response is usable by all aircraft in view because the pseudo interrogator sets up a pseudorandom sequence that can be used to identify it and to time its transmission and arrival times. This eliminates one of the main problems with the previous method. Moreover, the power consumption on the aircraft is significantly reduced due to the passivity of the system. However, since the method relies on calculating a pseudorange from the response using the broadcast time, it requires good absolute time at the pseudo interrogator. In addition, unlike in the previous method, an upgrade to the existing DME network in the form of the pseudo interrogators is needed. The DME-based passived ranging method is visualized in Fig. 1 by the second item, in which the aircraft interrogation is replaced by the blue arrow on the left, representing the pseudo interrogation.
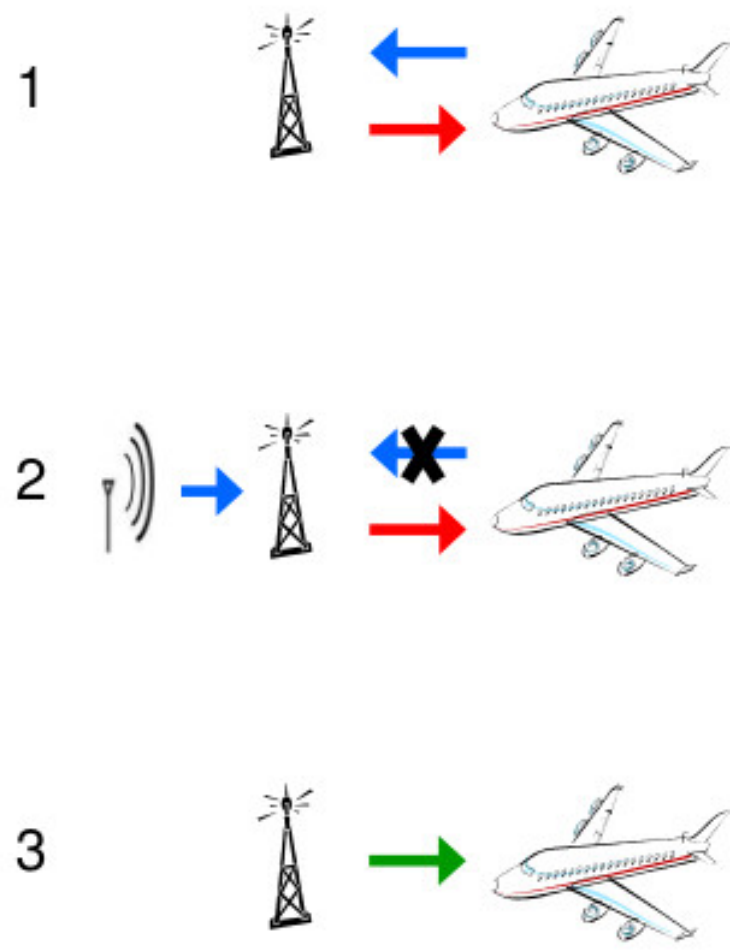

Figure 1: Proposed APNT methods using DME

This paper presents a different, novel method to estimate absolute aircraft position and velocity. The proposed method is based on accumulated delta range measurements from DME ground transponders using existing DME "heartbeat" signals. These measurements are provided by radio-navigation carrier phase observables of the "heartbeat" broadcasts.
A DME transponder naturally broadcasts its "heartbeat" signals even without an aircraft interrogation. Phase coherency of these signals, despite their non-continuous nature, is required for this method. The underlying carrier phase has been shown to be continuous for most transponders in service [3]. The intermittent nature of the "heartbeat" signals requires a special phase lock loop (PLL) to extract this continuous carrier phase. Using such a special PLL, it has been demonstrated that the carrier phase is trackable to $0.02 \mathrm{~m}$ standard deviation accuracy or better as though it is continuously available [4]. DME carrier phase has been used to smooth absolute range measurements and to provide position continuity during outage gaps of absolute range availability [5]. The new concept presented in this paper is the derivation of absolute information from the accumulated delta range data in addition to information about the relative time evolution of position.

This new APNT method has two advantages. First, it requires few upgrades to DME ground transponders, if any. The only needed features are phase coherency, as discussed above, and an adequate frequency standard. Unlike the DME-based passive ranging method, the proposed method requires no transfer of absolute time at the transponders. The second advantage of the method is the complete passivity of this system. The lack of DME interrogation prevents DME transponder overload with increasing air traffic. Furthermore, this passivity allows flight units to rely only on a DME-band receiver, as opposed to an active DME unit, and the accompanying reduction in required power makes this method practical for small aircraft.

Item 3 in Fig. 1 demonstrates the lack of DME interrogation by an absence of the blue arrow while the reliance on the ground transponder "heartbeat" signals is represented by the green arrow.

The proposed method is similar to Doppler-based radionavigation, especially as used for spacecraft orbit determination. The shapes of the accumulated delta range time histories are dependent on the absolute aircraft position and motion relative to the DME ground transponder. As such, this method requires the aircraft to have non-zero velocity. Furthermore, the availability of a pressure altimeter is assumed in order to ensure vertical position observability. With sufficient geometric diversity in the DME sources, a full navigation solution can be obtained through proper filtering of a time series of data.

This paper conducts a simulation study of the proposed system with a medium-fidelity truth-model simulation and a corresponding many-state filter. Its goals are to demonstrate absolute position observability and to deduce likely filter convergence times and steady state accuracies for representative scenarios. This study seeks to determine whether the proposed method merits further study. Specif- 
ically, the convergence time to a $0.025 \mathrm{~nm}$ error standard deviation in latitude and longitude is investigated.

The rest of the paper is organized as follows. Section 2 provides a detailed description of the dynamics and measurement model of the filter and truth-model simulation developed in this paper. Section 3 provides additional details about the truth-model simulation. Section 4 presents the results of applying the filter to the simulated data. The paper's summary and conclusions are presented in Section 5.

\section{AIRCRAFT AND DME MODEL}

This section develops the state vector, the dynamics model, and the measurement model that are used by the truthmodel simulation and by the corresponding filter.

\subsection{State Vector}

Given $N$ number of DME ground stations in view, the state vector has dimension $(11+2 N)$. It is

$$
\underline{x}=\left[\begin{array}{c}
\phi \\
\lambda \\
h \\
\dot{\phi} \\
\dot{\lambda} \\
\dot{h} \\
\ddot{\phi} \\
\ddot{\lambda} \\
\ddot{h} \\
\dot{\delta}_{R} \\
\Delta h \\
\dot{\delta}_{1} \\
\vdots \\
\dot{\delta}_{N} \\
\beta_{1} \\
\vdots \\
\beta_{N}
\end{array}\right]
$$

The navigation filter state vector consists of the aircraft flight states, the aircraft unit clock frequency error state, and the pressure altimeter bias state. The state vector also includes a clock frequency error state and an accumulated delta range bias state for each DME ground station in view.

The first 9 states represent the aircraft flight information. $\phi, \lambda$, and $h$ are the aircraft position states in latitude, longitude, and altitude, in the WGS- 84 coordinate system. $\dot{\phi}, \dot{\lambda}$, and $\dot{h}$ are the aircraft velocity states in the same WGS-84 system. Finally, $\ddot{\phi}, \ddot{\lambda}$, and $\ddot{h}$ are the aircraft acceleration states in the same WGS-84 system.

An alternative and equally applicable approach, not considered for this paper, is to represent the aircraft position, velocity, and acceleration using an Earth-Centered, EarthFixed Cartesian coordinate system.

The $10^{\text {th }}$ state, $\dot{\delta}_{R}$, is the aircraft flight unit clock frequency error.

The $11^{\text {th }}$ state, $\Delta h$, is the pressure altimeter bias.

States 12 through $(11+N)$ are the DME ground station clock frequency errors.

States $(12+N)$ through $(11+2 N)$ are the DME accumulated delta range biases. As discussed in Section 1, the proposed method does not require absolute timing. Therefore, the state vector does not have clock phase errors as distinct states; instead, these delta range biases incorporate the clock phase errors in a manner that accurately represents their effects on the carrier-phase-based accumulated delta range measurements.

\subsection{Aircraft Dynamics}

The aircraft discrete-time dynamic propagation model is a simple linear kinematic model. It can be represented in the matrix form:

$$
\begin{aligned}
{\left[\begin{array}{c}
\phi \\
\lambda \\
h \\
\dot{\phi} \\
\dot{\lambda} \\
\dot{h} \\
\ddot{\phi} \\
\ddot{\lambda} \\
\ddot{h}
\end{array}\right]_{k+1}=\left[\begin{array}{ccc}
I_{3} & \Delta t * I_{3} & 0.5 \Delta t^{2} * I_{3} \\
0 & I_{3} & \Delta t * I_{3} \\
0 & 0 & I_{3}
\end{array}\right]\left[\begin{array}{c}
\phi \\
\lambda \\
h \\
\dot{\phi} \\
\dot{\lambda} \\
\dot{h} \\
\ddot{\phi} \\
\ddot{\lambda} \\
\ddot{h}
\end{array}\right]_{k} } \\
+\left[\begin{array}{c}
0.5 \Delta t^{2} * I_{3} \\
\Delta t * I_{3} \\
I_{3}
\end{array}\right] \underline{v}_{a k} \\
t_{k}=k \cdot \Delta t
\end{aligned}
$$

In Eq. (2), $I_{3}$ is the 3 -by-3 identity matrix, and 0 is a 3 -by-3 matrix with all zero entries. $\Delta t$ is the time between each filter step, and $k$ is the sample index. Eq. (3) defines the discrete sample time.

The state transition matrix represents the simple integration of aircraft position, velocity, and acceleration over one time step. $\underline{v}_{a k}$ is the 3-by-1 integrated white-noise random jerk. This term is an increment to the acceleration, thereby caus- 
ing the acceleration to be a random walk. $\underline{v}_{a k}$ has zero time correlation, has a zero mean, and has individual elements with zero cross-correlation.

\subsection{Clock/Bias Dynamics}

A 2-state model for each clock is assumed. This model is taken from [6]. It includes frequency random walk and additional random-walk phase noise. After re-packaging to be consistent with the lumping of clock phase errors into accumulated delta range biases, the clock/bias models take the form

$$
\begin{aligned}
{\left[\begin{array}{c}
\beta_{i} \\
\dot{\delta}_{i}
\end{array}\right]_{k+1}=} & {\left[\begin{array}{ccc}
1 & -\Delta t & \Delta t \\
0 & 1 & 0
\end{array}\right]\left[\begin{array}{c}
\beta_{i} \\
\dot{\delta}_{i} \\
\dot{\delta}_{R}
\end{array}\right]_{k} } \\
& +\left[\begin{array}{ccc}
-1 & 0 & 1 \\
0 & 1 & 0
\end{array}\right]\left[\begin{array}{c}
v_{p, i} \\
v_{f, i} \\
v_{p, R}
\end{array}\right]_{k} \\
& \text { for } i=1, \ldots, \mathrm{N} . \\
\dot{\delta}_{R_{k+1}}= & \dot{\delta}_{R_{k}}+v_{f, R_{k}}
\end{aligned}
$$

The quantities $v_{p, i}$ and $v_{f, i}$ are the correlated clock phase and frequency random noise for the $i^{\text {th }} \mathrm{DME}$ ground transponder clock. The quantities $v_{p, R}$ and $v_{f, R}$ are the correlated clock phase and frequency random noise for the aircraft DME receiver clock.

The correlated phase and frequency random noise for both the transponder clocks and the receiver clock have zero mean. Their covariances depend on the time step, $\Delta t$, the minimum root Allan variance of the clock, $\sigma_{\text {Allan }}$, and the duration at which the minimum root Allan variance applies, $\tau_{\min }[6]$.

Eq. (4) applies to each transponder clock separately. As discussed before, the bias state includes the clock phase information; therefore, the phase random noise of both the transponder clock and the receiver clock has an effect on the propagation of $\beta_{i}$.

Because the bias state only holds the information about the relative phase error between the receiver clock and the transponder clock, the phase noise difference, $v_{p, R}-v_{p, i}$, matters. A positive transponder clock phase noise reduces the bias and a positive receiver clock phase noise increases the bias. In a similar manner, the frequency error difference, $\dot{\delta}_{R}-\dot{\delta}_{i}$, enters into the propagation equation.

Eq. (5) represents the random walk of the aircraft unit clock frequency error.

\subsection{Altimeter Dynamics}

The pressure altimeter bias is assumed to be a constant. It has a simple dynamic propagation equation:

$$
\Delta h_{k+1}=\Delta h_{k}
$$

\subsection{Measurement Model}

The measurement vector consists of an accumulated delta range measurement for each DME ground station along with the pressure altimeter measurement:

$$
\underline{z}_{k+1}=\left[\begin{array}{c}
z_{\text {phase }_{i}} \\
\vdots \\
z_{\text {phase }_{N}} \\
z_{h}
\end{array}\right]
$$

Each accumulated delta range measurement is given by the following equations:

$$
\begin{aligned}
z_{\text {phase }_{i}}=\lambda \cdot \phi_{i_{k+1}}= & \rho_{i_{k+1}}+c \delta_{R_{k+1}}-c \delta_{i_{k+1}} \\
& +c \alpha_{i}+w_{i_{k+1}} \\
= & \rho_{i_{k+1}}+c \beta_{i_{k+1}}+w_{i_{k+1}}
\end{aligned}
$$$$
\text { where } \quad c \beta_{i_{k+1}}=c \delta_{R_{k+1}}-c \delta_{i_{k+1}}+c \alpha_{i}
$$

The quantity $\alpha_{i}$ is the constant accumulated delta range bias. Eq. (8) includes this constant bias, as well as the DME and aircraft unit clock phase errors, $c \delta_{i_{k+1}}$ and $c \delta_{R_{k+1}}$. Eq. (9) describes the relation between the constant accumulated delta range bias, $\alpha_{i}$, and the time-varying accumulated delta range bias that includes the clock error effects, $\beta_{i_{k+1}}$. The final line of Eq. (8) represents the actual DME accumulated delta range model used in the filter.

The quantity $\rho_{i_{k+1}}$ is the distance between the aircraft and the $i^{\text {th }} \mathrm{DME}$ ground station. This term is the only nonlinear term in the entire filter model.

The quantity $w_{i_{k+1}}$ is the discrete-time zero-mean, Gaussian white noise in the accumulated delta range measurement for the $i^{t h}$ DME ground transponder.

The last measurement is from the pressure altimeter:

$$
z_{h_{k+1}}=h_{k+1}+\Delta h_{k+1}+w_{h_{k+1}}
$$

The quantity $w_{h_{k+1}}$ is the discrete-time zero-mean, Gaus- 
sian white noise on the pressure altimeter measurement. Thus, the altimeter measurement model includes both an unknown bias and this high-frequency random noise component.

\section{TRUTH-MODEL SIMULATION}

A medium-fidelity truth-model simulation is used to obtain the measurement data necessary for assessment of the proposed filter.

Three different sets of DME ground stations are considered. These are shown in Fig. 2. The magenta plus signs are the DME stations of the New York State (NYS) region. The NYS region is considered to be the nominal case for this study. It has good DME ground station density and good geometric diversity of the direction vectors from the visible DME ground transponders to any aircraft flying near its center. The black plus signs are the DME ground stations of the Montana/Dakota region. This second region has been chosen to study the effects of low DME ground station density. In the truth-model simulation, a flight path that traverses the interior of the given region is considered for both of these regions.

The last region, the Mid/South Atlantic Seaboard region, is the one whose DME ground transponders are indicated by cyan plus signs. It has been chosen in order to study the effects of insufficient geometric diversity. This region is used to study how the system works while the aircraft is over the Atlantic and, therefore, all visible ground stations are to the west of the aircraft. A flight path that keeps the aircraft approximately $100 \mathrm{~km}$ from the coast is studied for this region.

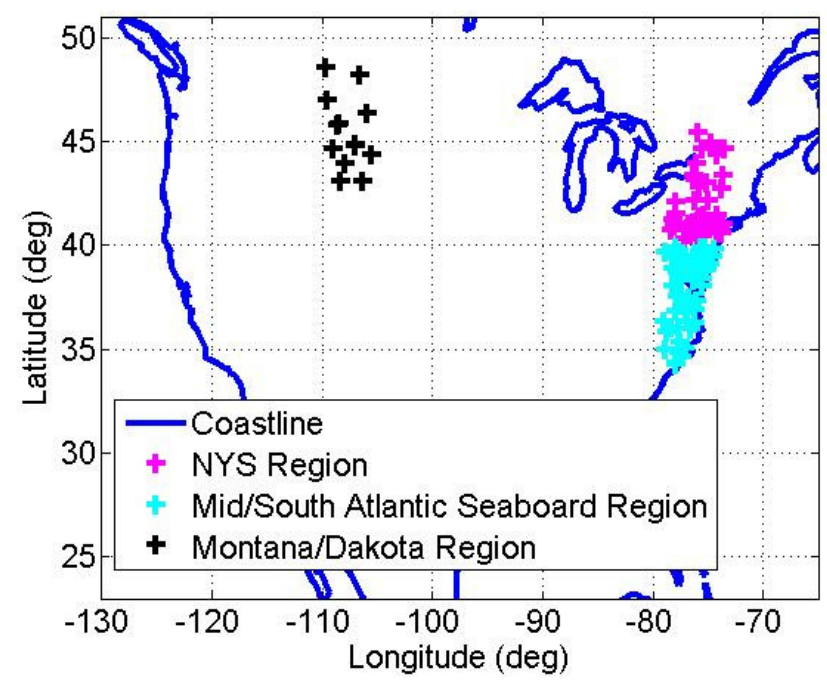

Figure 2: DME ground transponder locations for 3 regions of APNT study
The ground station locations that have been used in the study are not actual DME or TACAN stations, but they are close representatives of DME and TACAN ground station distributions in these areas.

The duration of the truth-model simulation is $1200 \mathrm{sec}-$ onds. A sampling frequency of $10 \mathrm{~Hz}$, with a corresponding $\Delta t$ of 0.1 seconds, has been used.

For each region, three sets of aircraft speeds (60, 200, and 500 knots) are considered. Furthermore, for each region/speed pair, three sets of aircraft altitudes are studied. Altitudes of 5000, 10000, and 30000 feet are chosen for the NYS and Mid/South Atlantic Seaboard regions. Because the average ground altitude in the Montana/Dakota region is 3000 feet, altitudes of 8000, 13000, and 30000 feet are instead studied for this region. This change puts the aircraft 5000 and 10000 feet above ground for the two low altitude cases, similar to the first two regions.

The initial state vector is propagated for the duration of the simulation using the state dynamics described in Section 2. This propagation includes the random effects of the process noise terms $\underline{v}_{a k}, v_{p, i}, v_{f, i}, v_{p, R}$, and $v_{f, R}$. These process noise terms are generated using a random number generator, and each individual simulation uses different samples of the process noise distributions, thus producing a slightly different actual trajectory through the filter's state space.

For each case, as indicated by the particular combination of the ground station region, the aircraft speed, and the aircraft altitude, 20 truth-model simulations have been run with the same initial trajectory, randomized initial filter errors, randomized process noise, and randomized measurement noise. The initial altitude rate and the initial acceleration states are both set to 0 for all cases.

Table 1 shows the clock model uncertainty parameters that are used for the truth-model simulation. The initial clock frequency error standard deviation, $\sigma_{f}$, the minimum root Allan variance, and the time interval over which this minimum applies, $\tau_{\min }$, are chosen to be consistent with the use of rubidium clocks for the DME ground station clocks and with an ovenized crystal oscillator for the aircraft receiver [7].

At each time step of the simulation, the expected measurement vector is calculated using the propagated state vector. A minimum/maximum DME signal range of 10/240 $\mathrm{km}$ has been applied to the simulation. This range limit is considered at each time step in order to assess whether the signal from a DME ground station can be realistically received by the aircraft receiver. Below the minimum range of $10 \mathrm{~km}$, an aircraft passes into a null of the DME ground transponder antenna. Above the maximum range of 240 $\mathrm{km}$, the signal becomes too weak to reliably track it. 
Table 1: Clock Standards

\begin{tabular}{cccc}
\hline Clock & $\sigma_{f}(\mathrm{~m} / \mathrm{sec})$ & Min. Root Allan Variance & $\tau_{\min }(\mathrm{sec})$ \\
\hline DME GS & 0.03 & $1.0 \mathrm{e}-13$ & 3000 \\
\hline Aircraft & 30 & $1.0 \mathrm{e}-11$ & 10 \\
\hline
\end{tabular}

In addition, the pathway of the signal from the DME ground station to the receiver is taken into account. Specifically, the simulation determines if the path of the signal pierces the 0 -altitude WGS-84 ellipsoid. At each time step, the phase measurements from only the DME ground stations that do not violate the range limit and the 0 -altitude piercing condition are included in the measurement vector. The pressure altimeter measurement is appended to the measurement vector after all the available DME measurements.

Respective standard deviations of 0.02 meters and 18 meters are chosen for the discrete-time Gaussian white noise on the accumulated delta range measurement and on the pressure altimeter measurement.

The integrated white-noise random jerk in the aircraft dynamics model has a standard deviation of $1 \mathrm{e}-11 \mathrm{rad} / \mathrm{sec}^{2}$, $1 \mathrm{e}-11 \mathrm{rad} / \mathrm{sec}^{2}$, and $5 \mathrm{e}-7 \mathrm{~m} / \mathrm{sec}^{2}$ for latitude, longitude, and altitude accelerations, respectively.

The pressure altimeter bias is sampled from a zero-mean, 18-m standard deviation Gaussian distribution for each of the 20 simulation runs.

\section{SRIF RESULTS WHEN APPLIED TO DATA FROM TRUTH-MODEL SIMULATIONS}

The Kalman filter algorithm developed in this paper uses the state, dynamics model, and measurement model discussed in Section 2. The Kalman filter is tested using the data from the truth-model simulation that is discussed in Section 3. The presented results have been generated using a Square Root Information (SRIF) implementation of an Extended Kalman filter. The reader is assumed to be familiar with this type of filter, but more information on Kalman filters can be found in [8] and more information on SRIF implementations is presented in [9].

Effectively, the EKF linearizes its nonlinear equations around the current state estimate using first-order Taylor series expansions. It then proceeds with the standard Kalman filter equations. The SRIF implementation is chosen for its numerical stability compared to the standard Kalman filter and for its capacity to represent zero a priori information on state parameters. This capability is needed for the new accumulated delta range bias state that is introduced every time a new DME ground transponder comes into view.
This study's proposed filter is adaptive in its state vector dimension. The filter is capable of adding and dropping states related to the DME ground stations as the signal from a DME ground station becomes available or goes out of range. Specifically, a clock frequency error state and an accumulated delta range bias state are added to the state before the very first Kalman filter measurement update for the ground station whose signal has just become available. The new ground transponder clock frequency error state is initialized at zero with the a priori standard deviation given in the left-most column of Table 1 . The new bias state is initialized with zero a priori information. All of the initial information about the new bias state will come entirely from the first accumulated delta range measurement from the ground station.

In a similar manner, the associated DME states are dropped off the state vector after the measurement update that contains the final measurement from a particular DME ground station.

Fig. 3 shows an example of the varying number of ground stations in view for a simulation case over the Montana/Dakota region at an altitude of $30000 \mathrm{ft}$ and a speed of $500 \mathrm{kts}$. Fig. 4 shows the corresponding variations in the state vector dimension.

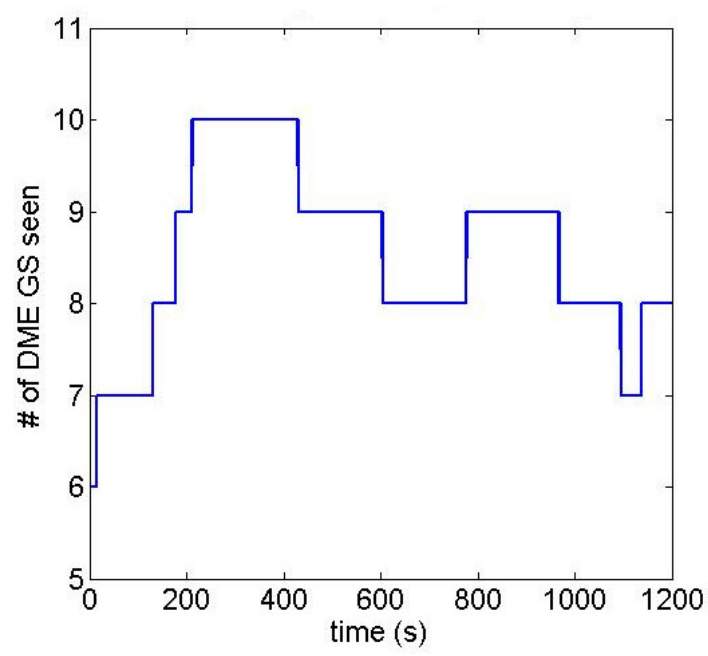

Figure 3: Time history of count of visible DME ground transponders for a simulation case over the Montana/Dakota region at an altitude of $30000 \mathrm{ft}$ and a speed of $500 \mathrm{kts}$

The filter is initialized with an aircraft position that has a 
$577 \mathrm{~m} \mathrm{1- \sigma}$ error in latitude, longitude, and altitude. The aircraft velocity is sampled with a $5.77 \mathrm{~m} / \mathrm{sec} 1-\sigma$ error in latitude, longitude, and altitude rates. The initial filter aircraft accelerations are set to 0 , while the respective latitude, longitude, and altitude acceleration error standard deviations are set to $0.064 \mathrm{~m} / \mathrm{sec}^{2}, 0.064 \mathrm{~m} / \mathrm{sec}^{2}$, and 0.1 $m / \sec ^{2}$.

For each case, the filter is applied to the 20 simulation runs, and the filter results are compared with the 20 true state histories from the truth-model simulation in order to carry out a statistical analysis of filter performance.

Figs. 5, 6, 7, and 8 are from an example case that flies through the Montana/Dakota region. The aircraft has a speed of $500 \mathrm{kts}$ and an altitude of $30000 \mathrm{ft}$.

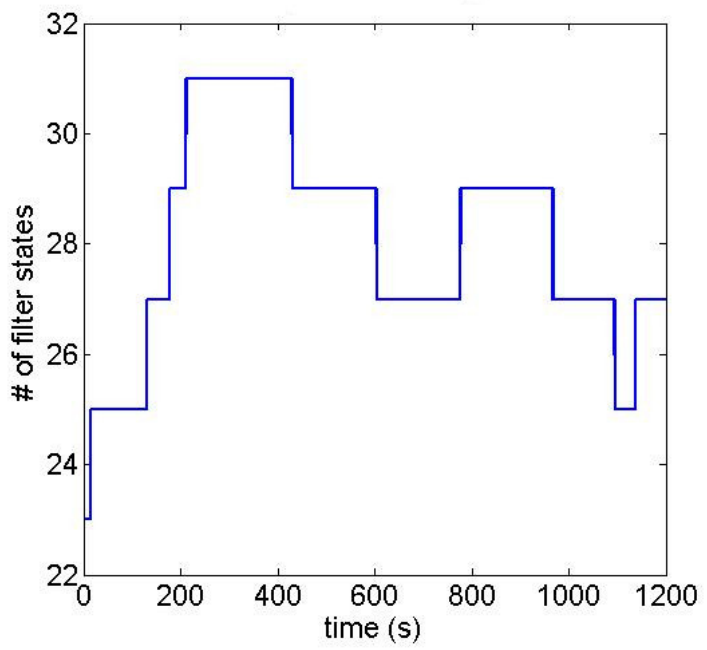

Figure 4: Time history of filter state dimension for a simulation case over the Montana/Dakota region at an altitude of $30000 \mathrm{ft}$ and a speed of $500 \mathrm{kts}$

Fig. 6 compares the state estimation error standard deviation time histories for the latitude, longitude, and altitude reported by the filter with those calculated by computing the 20-simulation-case statistics of the differences between the estimated filter position components and the simulated truth values of these components. The vertical axis for the latitude and longitude plots uses a logarithmic scale. For all three position states, the standard deviation reported by the filter (blue dashed lines) matches fairly well with the true error standard deviation (red solid lines).

Figs. 7 and 8 show the filter convergence for the clock frequency error states. The filter is able to bring the uncertainty in the clock frequency states down to $0.01 \mathrm{~m} / \mathrm{sec}$ accuracy within the duration of the experiment, for both the aircraft clock (Fig. 7) and a representative DME clock (Fig. 8).

A summary of all the filter results for the NYS region is

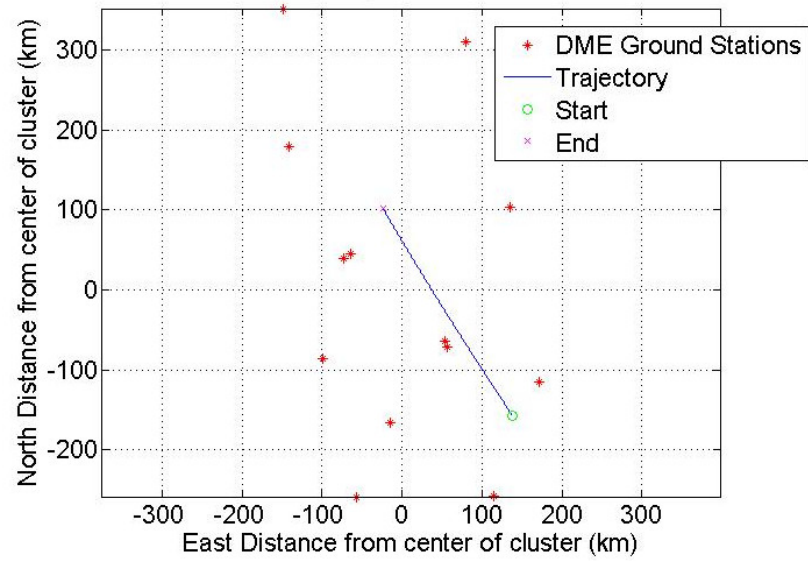

Figure 5: Plan view of an example simulated aircraft trajectory for a simulation case over the Montana/Dakota region at an altitude of $30000 \mathrm{ft}$ and a speed of $500 \mathrm{kts}$

presented in Table 2. The first and third columns define the case altitude/speed combination. The second column, $N_{\text {avg }}$, indicates the average number of ground stations in view for the duration of the simulation. The fourth column presents the time when the filter has converged to a navigation solution in latitude and longitude with a $1-\sigma$ accuracy of 0.025 nautical miles $(46.3 \mathrm{~m})$. This number is obtained from the top two panels of Fig. 6, by determining the intersection of the $46.3 \mathrm{~m}$ goal (green dashed lines) and the true error standard deviation (red solid lines). The greater number from the two panels is reported as the convergence time. This convergence time yields a $95 \%$ confidence that the latitude and longitude error is no larger than $0.05 \mathrm{~nm}$, which is a sufficient accuracy to meet the RNAV 0.1 requirement.

The fifth, sixth, and seventh columns are the steady state $1-\sigma$ accuracies achieved by the filter in latitude, longitude, and altitude, from left to right.

The filter has managed to converge to the $0.025 \mathrm{~nm}$ horizontal accuracy within the simulation duration in all cases. Moreover, the steady state horizontal accuracy values are much better than the 0.025 nautical mile goal.

The results indicate that the altitude does not have a significant effect on filter convergence. The aircraft speed, however, noticeably alters both the convergence time and the steady state accuracy. In particular, the cases with a speed of $500 \mathrm{kts}$ show the best results, while the cases with a speed of $60 \mathrm{kts}$ give the worst results. This is in line with the nature of the accumulated delta range time histories. A higher speed improves the observability for the system, and these results confirm this expectation.

A summary of the filter results for the Montana/Dakota region is presented in Table 3. The column structure is the same as in Table 2. This region has an overall fewer num- 
Table 2: Summary of Filter Results for NYS Region

\begin{tabular}{|c|c|c|c|c|c|c|}
\hline Altitude (ft) & $N_{\text {avg }}$ & Speed (kt) & $\begin{array}{l}\text { Convergence } \\
\text { Time to } 0.025 \\
\text { nm lat/long (s) }\end{array}$ & $\sigma_{\text {Lat }, s s}(\mathrm{~m})$ & $\sigma_{\text {Long,ss }}(\mathrm{m})$ & $\sigma_{a l t, s s}(\mathrm{~m})$ \\
\hline & \multirow{3}{*}{19} & 60 & 760 & 16 & 13 & 120 \\
\hline \multirow{3}{*}{5000} & & 200 & 225 & 0.3 & 1.6 & 25 \\
\hline & & 500 & 120 & 0.1 & 0.7 & 15 \\
\hline & \multirow{3}{*}{25} & 60 & 880 & 11 & 22 & 173 \\
\hline \multirow[t]{2}{*}{10000} & & 200 & 290 & 0.2 & 1.2 & 18 \\
\hline & & 500 & 60 & 0.06 & 0.2 & 1 \\
\hline \multirow{3}{*}{30000} & \multirow{3}{*}{27} & 60 & 898 & 15 & 15 & 56 \\
\hline & & 200 & 267 & 0.3 & 0.6 & 3 \\
\hline & & 500 & 80 & 0.05 & 0.1 & 0.6 \\
\hline
\end{tabular}
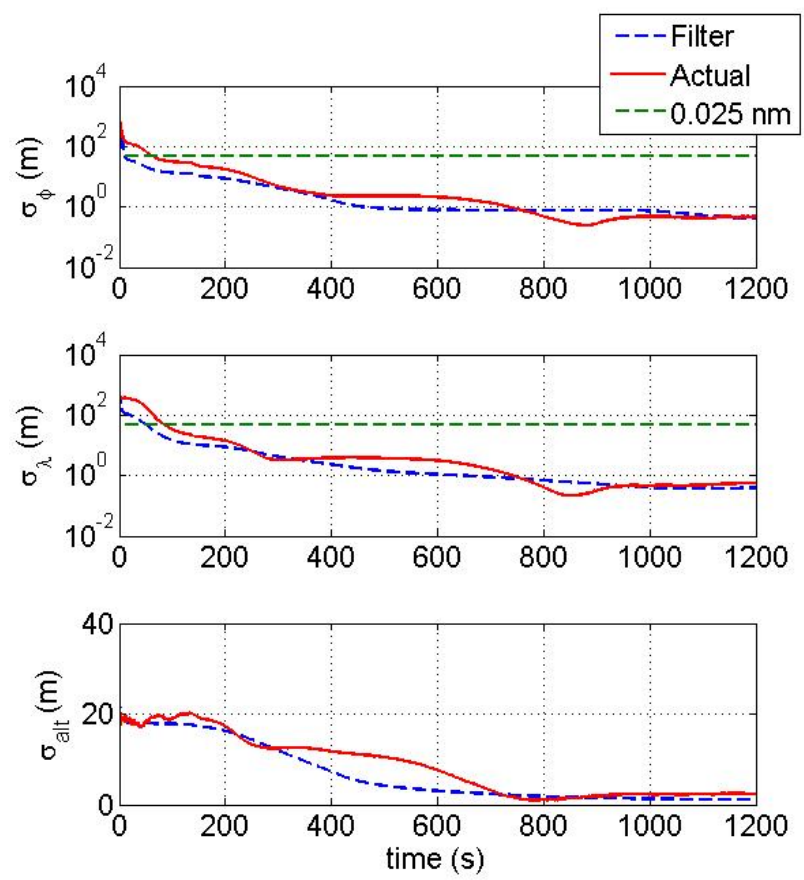

Figure 6: Latitude, longitude, and altitude position error standard deviation time histories for the cases in the Montana/Dakota region at an altitude of $30000 \mathrm{ft}$ and a speed of $500 \mathrm{kts}$

ber of ground stations in view for all cases. The symbol "-" in the convergence time column represents a failure to converge to lateral error standard deviations below $0.025 \mathrm{~nm}$ in the simulation duration of 1200 seconds. The symbol "*" attached to a number in the steady state accuracy columns indicates that this number is not a steady state value in reality, but rather the value the filter reached at the end of the simulation duration.

Unlike NYS region, where the filter has managed to converge in all cases, in Montana/Dakota region the filter has failed to converge for the cases with a speed of $60 \mathrm{kts}$.

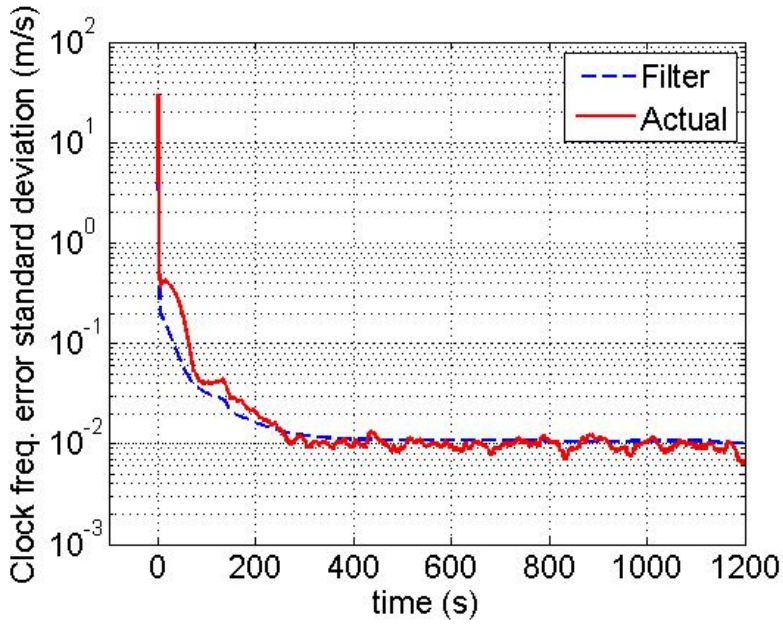

Figure 7: Airborne receiver clock frequency error standard deviation time histories for the cases in the Montana/Dakota region at an altitude of $30000 \mathrm{ft}$ and a speed of $500 \mathrm{kts}$

Moreover, the filter has slower convergence times and reduced accuracy. However, for all the cases in which the filter does indeed converge, the steady state accuracy values are again significantly below the 0.025 nautical miles goal.

In order to assess the impact of the initial DME clock frequency error uncertainty on the 60 knot cases, an additional case has been investigated. The $60 \mathrm{knot}, 8000$ foot case is repeated with initial DME clock frequency error $1-\sigma$ values of $0.003 \mathrm{~m} / \mathrm{sec}$ instead of $0.03 \mathrm{~m} / \mathrm{sec}$. Its results are reported in italics in Table 3 . This outcome shows improvement in the $\sigma$ accuracy values; however, the filter still fails to converge to horizontal error standard deviations below $0.025 \mathrm{~nm}$ within the $1200 \mathrm{sec}$ simulation window.

A summary of the filter results for the Mid/South Atlantic Seaboard region is presented in Table 4. The column struc- 
Table 3: Summary of Filter Results for Montana/Dakota Region

\begin{tabular}{|c|c|c|c|c|c|c|}
\hline Altitude (ft) & $N_{\text {avg }}$ & Speed (kt) & $\begin{array}{l}\text { Convergence } \\
\text { Time to } 0.025 \\
\mathrm{~nm} \text { lat/long (s) }\end{array}$ & $\sigma_{L a t, s s}(\mathrm{~m})$ & $\sigma_{\text {Long }, s s}(\mathrm{~m})$ & $\sigma_{\text {alt }, s s}(\mathrm{~m})$ \\
\hline \multirow{4}{*}{8000} & \multirow{4}{*}{8} & 60 & - & $75^{*}$ & $85^{*}$ & 21 \\
\hline & & 60 & - & 29 & $65^{*}$ & 18 \\
\hline & & 200 & 428 & 1.4 & 2.3 & 30 \\
\hline & & 500 & 60 & 0.3 & 0.3 & 5 \\
\hline \multirow{3}{*}{13000} & \multirow{3}{*}{9} & 60 & - & $39^{*}$ & $73^{*}$ & 19 \\
\hline & & 200 & 506 & 2.1 & 3.5 & 28 \\
\hline & & 500 & 203 & 0.7 & 0.6 & 7 \\
\hline \multirow{3}{*}{30000} & \multirow{3}{*}{9} & 60 & - & $63^{*}$ & $90^{*}$ & 16 \\
\hline & & 200 & 450 & 3.1 & 5 & 14 \\
\hline & & 500 & 84 & 0.8 & 0.5 & 2.2 \\
\hline
\end{tabular}

ture is the same as in Table 2. The symbols "-" and "*" have the same meaning as in Table 3 .

The $60 \mathrm{kts}$ cases have failed to converge, similar to the Montana/Dakota region. This region presents another failed convergence case, with a speed of $200 \mathrm{kts}$ and an altitude of 5000 feet. This case has been repeated with initial DME clock frequency error $1-\sigma$ values of $0.003 \mathrm{~m} / \mathrm{sec}$ instead of $0.03 \mathrm{~m} / \mathrm{sec}$. The results, reported in italics, show that a lower initial DME clock frequency error uncertainty leads to an actual convergence, unlike the attempt at $60 \mathrm{kts}$ in the Montana/Dakota region.

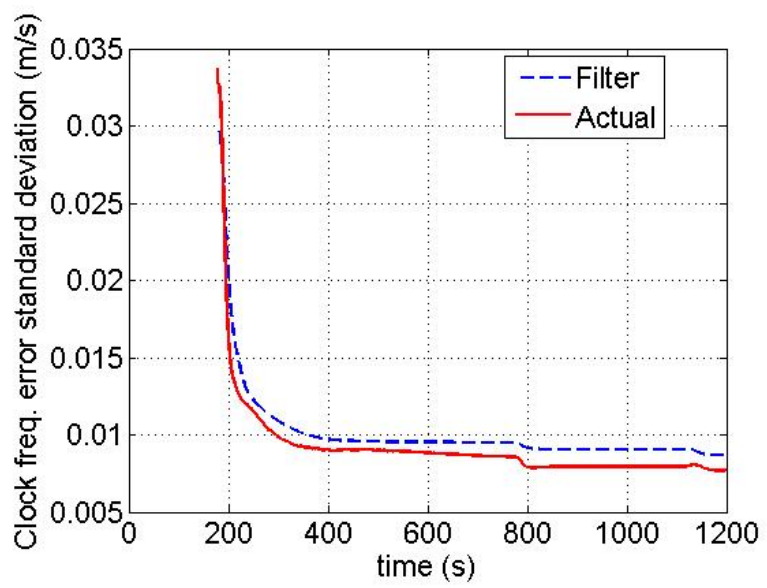

Figure 8: Representative DME ground transponder clock frequency error standard deviation time histories for the cases in the Montana/Dakota region at an altitude of 30000 $\mathrm{ft}$ and $a$ speed of $500 \mathrm{kts}$

As expected, the insufficient DME geometric diversity in this region leads to inferior results both in convergence times and in the steady state accuracy values. However, again, in the converged cases, the steady state horizontal accuracy values are significantly better than the 0.025 nautical mile goal.

\section{SUMMARY AND CONCLUSIONS}

This paper has investigated a new method for APNT based on DME carrier phase. An SRIF implementation of an Extended Kalman filter has been developed, and a Monte Carlo truth-model simulation has been conducted to assess the likely performance of this proposed APNT method.

The results suggest that, given enough time, a sufficient aircraft speed, and a reasonable number of visible ground stations, the SRIF filter is able to achieve convergence. Furthermore, the steady state accuracy observed in the converged cases is not only within useful range for APNT, defined as 0.025 nautical mile $1 \sigma$ error in order to satisfy RNAV 0.1 requirements, but also an order of magnitude below this threshold in many cases.

The cases that have been considered indicate that altitude has a relatively minor effect on filter performance, and they confirm that ground station density and geometric diversity together allow faster filter convergence. In addition, an increase in aircraft speed improves both the convergence speed of the position estimate and its steady state accuracy.

The accuracy levels demonstrated by this simulation study suggest potential usefulness of the proposed method as part of an APNT solution.

\section{REFERENCES}

[1] Narins, M., Enge, P., Peterson, B., Lo, S., Chen, Y.H., Akos, D., and Lombardi, M., "The Need for a Robust Precise Time and Frequency Alternative to Global Navigation Satellite Systems", Proc. ION GNSS 2012, Nashville, TN, Sept. 17-21, 2012, pp. 2057-2062.

[2] Lo, S., Peterson, B., Akos, D., Narins, M., Loh, R., and Enge, P., "Alternative Position Navigation \& Tim- 
Table 4: Summary of Filter Results for Mid/South Atlantic Seaboard Region

\begin{tabular}{|c|c|c|c|c|c|c|}
\hline Altitude (ft) & $N_{\text {avg }}$ & Speed (kt) & $\begin{array}{l}\text { Convergence } \\
\text { Time to } 0.025 \\
\text { nm lat/long (s) }\end{array}$ & $\sigma_{\text {Lat }, s s}(\mathrm{~m})$ & $\sigma_{\text {Long,ss }}(\mathrm{m})$ & $\sigma_{a l t, s s}(\mathrm{~m})$ \\
\hline \multirow{4}{*}{5000} & \multirow{4}{*}{5} & 60 & - & 473 & 250 & 24 \\
\hline & & 200 & - & $91^{*}$ & 38 & 11 \\
\hline & & 200 & 426 & 9 & 15 & 16 \\
\hline & & 500 & 464 & 3.8 & 11 & 19 \\
\hline \multirow{3}{*}{10000} & \multirow{3}{*}{10} & 60 & - & $247^{*}$ & $207^{*}$ & 20 \\
\hline & & 200 & 977 & 8 & 6 & 20 \\
\hline & & 500 & 420 & 1 & 2 & 22 \\
\hline \multirow{3}{*}{30000} & \multirow{3}{*}{12} & 60 & - & $159^{*}$ & $157^{*}$ & 26 \\
\hline & & 200 & 860 & 4 & 4 & 30 \\
\hline & & 500 & 371 & 0.7 & 1.4 & 10 \\
\hline
\end{tabular}

ing (APNT) Based on Existing DME and UAT Ground Signals", Proc. ION GNSS 2011, Portland, OR, Sept. 19-23, 2011, pp. 3309-3317.

[3] Li, K., and Pelgrum, W., "Flight Test Evaluation of Enhanced DME (eDME) Performance Enhancements", Proc. ION GNSS 2012, Nashville, TN, Sept. 17-21, 2012, pp. 803-811.

[4] Li, K., and Pelgrum, W., "Robust DME Carrier Phase Tracking Under Flight Dynamics", Proc. ION ITM 2013, San Diego, CA, Jan. 27-30, 2013, pp. 696-708.

[5] Naab-Levy, A., Li, K., and Pelgrum, W., "DME/N Error Budget Allocation and DME-Next Proof-ofConcept Flight Test and Performance Evaluation", Proc. ION Pacific PNT 2013, Honolulu, HI, April 2325, 2013, pp. 434-450.

[6] Brown, R. G. and Hwang, P. Y. C., Introduction to Random Signals and Applied Kalman Filtering, Wiley, New York, 3rd ed., Nov. 1997, pp. 428-432.

[7] Vig, J.R., "Introduction to Quartz Frequency Standards", Report No. SLCET-TR-92-1, US Army Laboratory Command, Electronics Technology and Devices Laboratory, March 1992.

[8] Bar-Shalom, Y., Li, R. X., and Kirubarajan, T., Estimation with Applications to Tracking and Navigation, John Wiley \& Sons, New York, NY, 2001.

[9] Bierman, G. J., Factorization Methods for Discrete Sequential Estimation, Academic, New York, NY, 1977. 